\title{
Preface to the special issue on Interdisciplines
}

\author{
YI Sheng-hui(易圣辉) ${ }^{1}$, ZHAOJin-jin(赵晋津 $)^{2}$ \\ 1. Centre for Advanced Structural Materials, City University of Hong Kong Shenzhen Research Institute, \\ Greater Bay Joint Division, Shenyang National Laboratory for Materials Science, Shenzhen 518057, China; \\ 2. Institute of Materials for Energy Conversion, School of Materials Science and Engineering, \\ Shijiazhuang Tiedao University, Shijiazhuang 050043, China \\ (C) Central South University Press and Springer-Verlag GmbH Germany, part of Springer Nature 2021
}

Interdisciplinary research is an inevitable trend in scientific development and an important driving force to accelerate scientific and technological innovations. How to promote interdisciplinary research has always been a common question and a challenge faced by science research funding agencies all over the world. The interdisciplinary research would strengthen the coordination and integration of engineering strategic needs and emerging frontiers of science. To respect the characteristics of different disciplines and break shackles of traditional concepts, we organized this special issue with the title of "Interdisciplines" focusing on the advances in developing new materials science and artificial intelligence in the field of interdisciplinary research in Journal of Central South University, to promote in-depth crossintegration, strive to form new growth points of disciplines, and train innovative interdisciplinary talents.

This special issue collects 22 contributions, including 3 reviews and 19 articles. The contributions cover various topics, such as nanoparticles, ceramic materials, optoelectronic materials, cement materials, human tissues, and other functional and structural materials. Also, the analysis algorithms and digital devices are involved for intelligent decision in our industrial and life. Review papers are focused on new materials and intelligent laser fabrication. The collected works explore the interdisciplinary among new materials, new methods, new functional, structural features and new phenomenon.

Firstly, focusing on health issues, LIN Xin et al investigated the effect of gold nanoparticles shape on neurophysiological properties of neurons, including nanosphere, nanotriakisoctahedron and nanoflower shapes, and found that the neurotoxicity of gold nanoparticles is surface-roughness dependent. ZHANG Chuan-kun et al investigated three kinds of chemical protective clothing to study the relationship among clothing thermal properties, physiological responses, and environmental conditions. The results showed that physiological responses were significantly affected by the clothing thermal features and activity intensity in hot-humid environment. NING Jing-heng et al tried to provide an economic and eco-friendly solution for the treatment of the dye wastewater by using the ferroferric oxide@ $\beta$-cyclodextrin to efficiently adsorb and remove the basic fuchsin. WANG Zilong et al fabricated a bifunctional gel polymer electrolyte with a good discharge capacity to tackle the lithium dendrites and the shuttle effect in lithium-sulfur batteries.

Secondly, focusing on inorganic materials, YANG Shuai et al proposed a novel ultra-highvoltage $4 \mathrm{H}$ polytype silicon carbide merged p-type/ intrinsic/n-type Schottky diode with 3D p-type buried layers for the next generation semiconductor chip, to overcome the forward conduction characteristic and the reverse leakage current. $\mathrm{HU}$ Yan-jun et al prepared $\mathrm{Al}_{2} \mathrm{O}_{3}$ ceramics by a low-cost pressureless sintering technology, using the binary 
sintering aids of $\mathrm{MgO}$ and $\mathrm{SiO}_{2}$ for promoting the densification and mechanical properties. CHENG Zheng-chun et al used shape-persistent polyphenylene dendritic $\mathrm{C}_{60}$ derivatives as the electron transport materials for perovskite solar cells and achieved a maximum power conversion efficiency of $14.7 \%$ taking advantage of the high solubility. FANG Li-mei et al studied $\mathrm{MnPSe}_{3} / \mathrm{WS}_{2}$ heterostructure with the first principle calculations by exploring its electronic properties under the application of an electric field and found that the photocatalytic performance could be modulated by adjusting the band edges and band gap. JIANG Haohong et al proposed a new hybrid interconnection layer with doping graphene oxide in all solutionprocessed red tandem quantum dot light-emitting diodes for flat panel display. A $21 \%$ growth and a $51 \%$ increase were found in the maximum luminance and current efficiency. FENG Chao et al proposed a series of new ceramics of perovskite structures with both high Curie temperature and large piezoelectric constant by taking the balance between rhombohedral and tetragonal phase near morphotropic phase boundary with dense microstructure of ceramics. A piezoelectric ceramic based high-temperature acoustic emission sensor was also fabricated of high performance. TAO Jixiao et al investigated the thermal postbuckling and nonlinear panel flutter motions of variable stiffness composite laminates and used piezoelectric actuators to optimally control their dynamic responses. FAN Bao-yan et al synthesized a catalyst of ferroelectric-BaTiO $@_{3} @$ photoelectric- $\mathrm{TiO}_{2}$ nanohybrids of mesoporous microstructures with enhanced photocatalytic activity. The intimate interfacial interactions are effective in driving the separation and transport of photogenerated charge carries. To capture the microscale humidity inside cement-based materials in situ, GU Hai-tao et al used rhodamine $6 \mathrm{G}$ to characterize the change in relative humidity in cement-based materials by its fluorescence intensity, which had little effect on the hydration heat release and hydration products of cement-based materials. Also, metallic materials are involved in this special issue. WANG Lin et al experimentally investigated the mechanical properties of an $\mathrm{Al}-\mathrm{Cu}-\mathrm{Li}$ alloy subjected to cryorolling or hot rolling and subsequent aging, focusing on microstructure evolution.

In the area of digital devices, MO Shuai et al proposed a brushless motor drive and control system for the robot joint for the micro-service robots and manipulators, which has high performance, low cost and easy operation. GUO Dong et al proposed a smartphone-based calibrationfree portable urinalysis device for screening tests or daily monitoring of various diseases, which can complete one test in $\sim 2 \mathrm{~min}$.

Focusing in the industrial area, HU Yun-yun et al proposed a fault degradation-oriented slow feature analysis algorithm to extract fault degradation directions using a key fault degradation factor and an online fault prognostic strategy, which tried to capture fault degradation information and model nonstationary processes in industrial processes. DONG Long-jun et al proposed a manmachine-environment system of evaluation indexes for clean and safer production of phosphorus mines. LI Xiao-jun et al reported the optimal setting form of visual fatigue relief zone, such as its length and location, using natural landscape decoration inside the tunnel to improve driving fatigue by integrating virtual reality apparatus with wearable electroencephalogram-based devices.

The review works involve femtosecond laser fabrication and new materials. ZHU Zhuo et al reviewed the recent research progress of femtosecond laser micro/nano fabrication for bioinspired superhydrophobic or underwater superoleophobic surfaces, which have wide applications in self-cleaning, drag reduction, oilwater separation, anti-oil pollution, etc. SUN Haodong et al reviewed the recent advances in organic triboluminescent materials and their applications in stress sensing, movement monitoring, imaging stress distribution, visualization of crack propagation, structural diagnosis, and other sensing fields. MXenes are emerging 2D nanomaterials composed of transition metal carbides and nitrides and possess unique layered structures with abundant surface functional groups, which are suitable for optoelectronic device applications. CHEN Ke-fan et al reviewed the mainstream synthesis methods, optical and electrical properties of MXenes and 
their applications in solar cells and light-emitting diodes.

In summary, these contributions present the current interdisciplinary researches from different aspects. We would like to sincerely thank all the coauthors who have made important contributions to this Special Issue. We also thank the professional editorial office of Journal of Central South University for their hard-works to make this issue such a great success.

YI Sheng-hui currently takes a position of senior research fellow in Center for Advanced Structural Materials in City University of Hong Kong Shenzhen Research Institute. He obtained his $\mathrm{PhD}$ from City University of Hong Kong in 2016, and his PhD supervisors are Prof. LU Jian and Prof. HE Xiao-qiao. His research interests include morphing structures, nano-metallic materials, residual stress and perovskite materials.

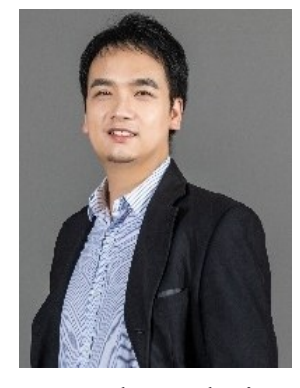

YI Sheng-hui

$\mathrm{PhD}$, Senior Research Fellow

E-mail: shenghyi2-c@my.cityu.edu.hk

ORCID: https://orcid.org/0000-0002-0499-3228
ZHAO Jin-jin is the director of Institute of Materials for Energy Conversion in Shijiazhuang Tiedao University. She obtained her $\mathrm{PhD}$ in materials physics and chemistry from Shanghai Institute of Ceramics Chinese Academy of Sciences (SICCAS) in 2010, and her PhD supervisors are Prof. SHI Jian-lin and Prof. CHEN Hang-rong. She did her visiting doctoral studies from Max Plank Institute of Colloids and Interfaces, Germany, from October 2007 to October 2008, and visiting scholar at the University of Washington from August 2015 to August 2016, and visited at City University of Hong Kong from July 2019 to October 2019. She held faculty appointment in Shijiazhuang Tiedao University. She is interested in interdisciplinary research on perovskite materials and devices.

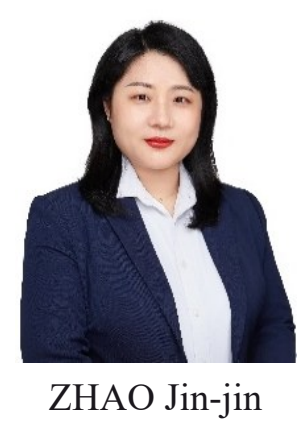

$\mathrm{PhD}$, Professor, Director of Institute of Materials for Energy Conversion E-mail: jinjinzhao2012@163.com ORCID: https://orcid.org/0000-0003-1620-3040 